\title{
A Populus TIR1 gene family survey reveals differential expression patterns and responses to 1-naphthaleneacetic acid and stress treatments
}

\section{OPEN ACCESS}

Edited by:

Ramin Yadegari,

The University of Arizona, USA

Reviewed by:

Gabriela Carolina Pagnussat, Universidad Nacional de Mar del Plata, Argentina David Selinger,

Pioneer Hi-Bred International, USA

*Correspondence: Shutang Zhao,

State Key Laboratory of Tree Genetics and Breeding, Research Institute of

Forestry, Chinese Academy of Forestry, Xiangshan Road, Beijing 100091, China zhaost318@163.com; Mengzhu Lu, Co-Innovation Center for Sustainable Forestry in Southern China, Nanjing

Forestry University, 159 Longpan Road, Nanjing, Jiangsu 210037, China lumz@caf.ac.cn

Specialty section: This article was submitted to Plant Genetics and Genomics, a section of the journa Frontiers in Plant Science

Received: 24 February 2015

Accepted: 20 August 2015

Published: 10 September 2015

Citation:

Shu W, Liu Y, Guo Y, Zhou H, Zhang J, Zhao $S$ and Lu M (2015) A Populus TIR1 gene family survey reveals differential expression patterns and responses to 1-naphthaleneacetic acid and stress treatments. Front. Plant Sci. 6:719. doi: 10.3389/fpls.2015.00719

\author{
Wenbo Shu ${ }^{1,2}$, Yingli Liu' ${ }^{2}$, Yinghua Guo ${ }^{2}$, Houjun Zhou ${ }^{2}$, Jin Zhang ${ }^{2}$, Shutang Zhao ${ }^{2 *}$ and \\ Mengzhu Lu ${ }^{1,2 *}$ \\ ${ }^{1}$ Co-Innovation Center for Sustainable Forestry in Southern China, Nanjing Forestry University, Nanjing, China, ${ }^{2}$ State Key \\ Laboratory of Tree Genetics and Breeding, Research Institute of Forestry, Chinese Academy of Forestry, Beijing, China
}

The plant hormone auxin is a central regulator of plant growth. TRANSPORT INHIBITOR RESPONSE 1/AUXIN SIGNALING F-BOX (TIR1/AFB) is a component of the E3 ubiquitin ligase complex SCF ${ }^{\mathrm{TIR} 1 / \mathrm{AFB}}$ and acts as an auxin co-receptor for nuclear auxin signaling. The SCF ${ }^{\mathrm{TR} 1 / \mathrm{AFB}}$-proteasome machinery plays a central regulatory role in development-related gene transcription. Populus trichocarpa, as a model tree, has a unique fast-growth trait to which auxin signaling may contribute. However, no systematic analyses of the genome organization, gene structure, and expression of TIR1-like genes have been undertaken in this woody model plant. In this study, we identified a total of eight TIR1 genes in the Populus genome that are phylogenetically clustered into four subgroups, PtrFBL1/PtrFBL2, PtrFBL3/PtrFBL4, PtrFBL5/PtrFBL6, and PtrFBL7/PtrFBL8, representing four paralogous pairs. In addition, the gene structure and motif composition were relatively conserved in each paralogous pair and all of the PtrFBL members were localized in the nucleus. Different sets of PtrFBLs were strongly expressed in the leaves, stems, roots, cambial zones, and immature xylem of Populus. Interestingly, PtrFBL1 and 7 were expressed mainly in vascular and cambial tissues, respectively, indicating their potential but different roles in wood formation. Furthermore, Populus FBLs responded differentially upon exposure to various stresses. Finally, over-expression studies indicated a role of FBL1 in poplar stem growth and response to drought stress. Collectively, these observations lay the foundation for further investigations into the potential roles of PtrFBL genes in tree growth and development.

Keywords: Populus, FBL family, phylogenetic analysis, expression, NAA, stress responses

\section{Introduction}

Plant growth and development are largely influenced by the surrounding environment, which is mediated by different phytohormones through signaling pathways. As a central regulator involved in nearly all aspects of plant development, auxin is synthesized by various plant tissues (Lau et al., 2009) and transported long-distance through vascular tissues or from cell to cell across 
membranes (Kramer and Bennett, 2006) to contribute to the robustness of the auxin signaling network (Peer, 2013). Within minutes, auxin can rapidly alter the expression of hundreds of genes, (Chapman and Estelle, 2009) which were classified into three major families: SMALL AUXIN-UP RNA (SAUR), GH3, and AUXIN/INDOLE-3-ACETIC ACID (Aux/IAA) genes. The SAUR genes encode unstable transcripts (Chapman and Estelle, 2009); the GH3 family encodes enzymes that catalyze the conjugation of IAA with an amino acid to yield an inactive storage form of IAA (Ludwig-Müller, 2011); and the Aux/IAA family plays a central role in auxin signal transduction that regulates the expression of auxin-responsive genes (Remington et al., 2004), which are involved in growth, development, and responses to abiotic stresses such as salt, drought, and temperature (Salehin et al., 2015).

The diversity in auxin perception is a key factor leading to the great variety of auxin responses (Woodward and Bartel, 2005). Three receptor/co-receptor complexes involved in auxin signaling have been identified: two inside of the cell [SCFTRANSPORT INHIBITOR RESPONSE 1/AUXIN SIGNALING F-BOX (TI

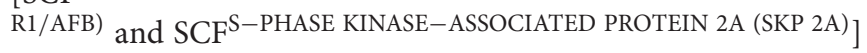
and one at the cell periphery, Auxin Binding Protein 1 (ABP1; Salehin et al., 2015). TIR1/AFB-Aux/IAA, the best characterized receptor system, regulates transcription of downstream auxinresponsive genes in the nucleus (Calderón Villalobos et al., 2012), while newly identified auxin receptors SKP2A and ABP1 mainly repress cell division during the cell cycle and subcellular protein trafficking, respectively (Jurado et al., 2008; Robert et al., 2010).

Nuclear auxin signaling involves TIR1/AFBs that is a component of the E3 ubiquitin ligase complex SCF ${ }^{\text {TIR1 }}$ (Gagne et al., 2002) and acts as an auxin co-receptor (Dharmasiri et al., 2005a,b). Auxin enhances the TIR1-Aux/IAA interaction by filling the bottom of a hydrophobic pocket in TIR1, which changes the properties of SCF ${ }^{\text {TIR1 }}$ (Yu et al., 2013). The SCF ${ }^{\mathrm{TIR} 1 / \mathrm{AFB}}$-proteasome machinery causes the proteolytic turnover of Aux/IAAs, thereby releasing ARFs and controlling the specificity of the downstream genes' transcription (Calderón Villalobos et al., 2012; Yu et al., 2013). Therefore, TIR1/AFB is the trigger that activates the auxin pathway.

Six Arabidopsis TIR1/AFB receptors, interacting with 29 Aux/IAA and 23 ARF combinations, modulate precisely the specific gene expression profile in response to auxin signaling (Salehin et al., 2015). TIR1 and AFB2 are positive regulators of auxin signaling (Parry et al., 2009), while AFB4, as a negative regulator, is epistatic to TIR1 and AFB2 (Greenham et al., 2011; Hu et al., 2012). TIR1 and AFB2 play more important roles during seedling development and root responses to the environment than either AFB1 or AFB3 (Parry et al., 2009). AFB3 has a unique role in the nitrate response of roots (Vidal et al., 2010), and AFB2 and AFB3 also have important roles in seed coat development (Locascio et al., 2014). AFB5 is part of an auxin receptor complex with a higher binding affinity for picloram (4-amino-3, 5, 6-trichloropicolinic acid) and specifically recognizes synthetic picolinate auxins (Walsh et al., 2006). TIR1/AFBs show different affinities for given Aux/IAAs (Parry et al., 2009; Calderón Villalobos et al., 2012) and the half-lives are significantly different among Aux/IAAs (Dreher et al., 2006). For a given Aux/IAA, TIR1, and AFB2 confer a more rapid degradation than AFB1 and AFB3 do (Havens et al., 2012). These results suggest different roles for TIR1/AFB in the regulation of auxin signaling, temporally, and spatially, under different stresses, such as salt (Chen et al., 2015) and drought (Guerra et al., 2015).

Woody plants have unique biological processes that are absent in model herbivorous plants, like Arabidopsis. For instance, continuous cylinder-like meristem is reactivated each year in woody plants (Fuchs et al., 2010). How the auxin signaling functions during these unique processes in woody plants remains to be elucidated. The recent sequencing of the Populus trichocarpa genome (Tuskan et al., 2006) has provided an opportunity to study the families of auxin signaling-related genes, such as Aux/IAA, ARF (Kalluri et al., 2007), WUSCHEL (WUS) (Liu et al., 2014a), and PIN-FORMED (PIN) (Liu et al., 2014b), laying the foundation for further investigations into the roles of auxin signaling in woody plant development. In this study, using Arabidopsis AtTIR1/AFB, we have identified PtrFBLs in the $P$. trichocarpa genome, and determined their structure, phylogenetic relationships and expression patterns. Eight PtrFBL members, as well as their expression profiles in different tissues and their responses under 1-naphthaleneacetic acid (NAA), heat, and drought treatments, are presented.

\section{Materials and Methods}

\section{Bioinformatics Analysis}

Published AtTIR1/AFB and PtrFBL gene sequences (Parry et al., 2009) were retrieved and used as queries in BLAST searches against the Arabidopsis and Populus genome databases (http://phytozome.jgi.doe.gov/pz/portal.html). Multiple fulllength sequences of AtTIR1/AFB and PtrFBL proteins were aligned using the CLUSTALX 2.0 software (Larkin et al., 2007). Un-rooted phylogenetic trees were constructed with MEGA 5 using the neighbor-joining method, with 1000 bootstrap replicates (Tamura et al., 2011). WoLF PSORT (http://www.genscript.com/psort/wolf_psort.html) was used to predict protein subcellular localizations. The isoelectric point and molecular weight were estimated using the Compute pI/Mw tool from ExPASy (http://web.expasy.org/compute_pi). A search for duplicated genes was performed using the Plant Genome Duplication Database (PGDD; http://chibba.agtec.uga. edu/duplication/). The chromosomal locations of the PtrFBL genes were determined using the Populus genome browser (http://phytozome.jgi.doe.gov/pz/portal.html\#! search? show= KEYWORD\&method=Org_Ptrichocarpa). The exon and intron structures of individual TIR1/AFB genes were illustrated using the Gene Structure Display Server (GSDS; http://gsds.cbi.pku. edu.cn/) (Hu et al., 2015) by aligning the cDNA sequences with the corresponding genomic DNA sequences.

Functional motifs or domains of TIR1/AFB and PtrFBL protein sequences were analyzed using PROSITE and the Conserved Domain database. MEME (http://meme.nbcr.net/ meme/) (Bailey et al., 2009) was used to identify motifs in candidate sequences with the following parameters: number 
of repetitions $=$ any, maximum number of motifs $=20$ and optimum motif widths constrained to from 30 to 70 residues.

\section{Plasmids and Constructs}

The coding sequences of PtrFBL1, 2, 3, 4, 5, 6, 7, and 8, without stop codons, were amplified from the cDNA of hybrid poplar $84 \mathrm{~K}$ (Populus alba $\times$ Populus glandulosa) and inserted into pEarleyGate101 (ABRC stock DB3$683)$ to produce the 35S::PtrFBL1-YFP, 35S::PtrFBL2-YFP, 35S::PtrFBL3-YFP, 35S::PtrFBL4-YFP, 35S::PtrFBL5-YFP, 35S::PtrFBL6-YFP, 35S::PtrFBL7-YFP, and 35S::PtrFBL8-YFP constructs, respectively, using the Gateway cloning system (Invitrogen). The approximately $2.5 \mathrm{~kb} \quad 5^{\prime}$-UTR fragments of PtrFBL1, PtrFBL4, PtrFBL5, and PtrFBL7 were amplified from the genomic DNA of $P$. trichocarpa Torr. The primer sequences for the promoters are listed in Table S1. The promoter fragments were then inserted into pDNOR222.1 and subcloned

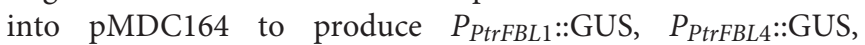
$P_{\text {PtrFBL5 }}:$ GUS, and $P_{\text {PtrFBL7 }}::$ GUS constructs using the Gateway cloning system (Invitrogen). The coding sequence of PtrFBL1 was amplified from the cDNA of $84 \mathrm{~K}$, cloned into pDNOR222.1 and sequenced. PtrFBL1 cDNA was further cloned into pMDC32 to produce 35S::PtrFBL1 constructs for transformation into poplar $84 \mathrm{~K}$. The transgenic poplar plants containing the auxin responsive promoter DR5 were obtained previously (Liu et al., 2014a). At least five independent transgenic lines were used for further analyses.

\section{Plant Cultivation and Transformation}

Tobacco (Nicotiana benthamiana) plants were cultivated under short-day photoperiod conditions ( $16 \mathrm{~h}$ light $/ 8 \mathrm{~h}$ dark). When the Agrobacterial culture reached the stationary growth phase at $28^{\circ} \mathrm{C}$ with agitation, cells were collected and resuspended in infiltration buffer $(100 \mu \mathrm{M}$ acetosyringone in $10 \mathrm{mM} \mathrm{MgCl} 2)$ to $\mathrm{OD}_{600}=0.8$. The leaves of 2-month-old tobacco seedlings expressing 35S::PtrFBLs-YFP were used for infiltration. The transient expression in lower leaf epidermal cells was performed as described by Liu et al. (2014a). After 5 to 7 days, the leaves were immersed in $50 \mu \mathrm{M} \mathrm{4}$, 6-diamidino-2-phenylindole (DAPI) for $60 \mathrm{~min}$ for a subcellular localization analysis. Fluorescence was observed using an UltraVIEW VoX 3D Live Cell Imaging System (PerkinElmer, USA). For imaging YFP and DAPI fluorescence, 488 and 405-nm excitations were used, respectively.

Hybrid poplar $(P$. alba $\times$ P. glandulosa) clone $84 \mathrm{~K}$ was used for stable transformations (Liu et al., 2014a). Histochemical GUS staining was performed by incubating 2 -week-old seedlings and 4 -week-old stem sections in $90 \%$ cold acetone. Each sample was washed three times with a staining buffer containing $50 \mathrm{mM}$ sodium phosphate ( $\mathrm{pH} 7.0$ ), $2 \mathrm{mM}$ potassium ferrocyanide, $2 \mathrm{mM}$ potassium ferricyanide, $10 \mathrm{mM}$ EDTA, and $0.2 \%(\mathrm{v} / \mathrm{v})$ Triton X100 on ice. The samples were then transferred into the staining solution [staining buffer with $20 \%(\mathrm{v} / \mathrm{v})$ methanol and $1 \mathrm{mM}$ $\mathrm{X}$-Gluc] and slowly subjected to vacuum infiltration, which penetrated the tissue with the staining solution. After a $12 \mathrm{~h}$ incubation at $37^{\circ} \mathrm{C}$ with $70 \mathrm{rpm}$ gentle agitation, the samples were rinsed in $70 \%$ ethanol for visual observation and microscopy.

\section{RNA Isolation, Semi-quantitative RT-PCR and qRT-PCR}

Total RNA was extracted from roots, leaves and young stems collected from young seedlings, cambial zones and immature xylem obtained by peeling the bark of 5-year-old poplar trees (Du and Groover, 2010) using the RNeasy Plant Mini Kit and treated with RNase-free DNase I (Qiagen, Hilden, Germany). Additionally, total RNA was extracted from hormoneand stress-treated seedlings collected at different times. Firststrand cDNA synthesis was carried out with approximately $3 \mu \mathrm{g}$ RNA using the Superscript III reverse transcription kit (Life Technologies, Carlsbad, CA, USA) according to the manufacturer's instruction. Specific 20-25 bp long RT-PCR primers with melting temperatures of $58-60^{\circ}$ Cand target lengths

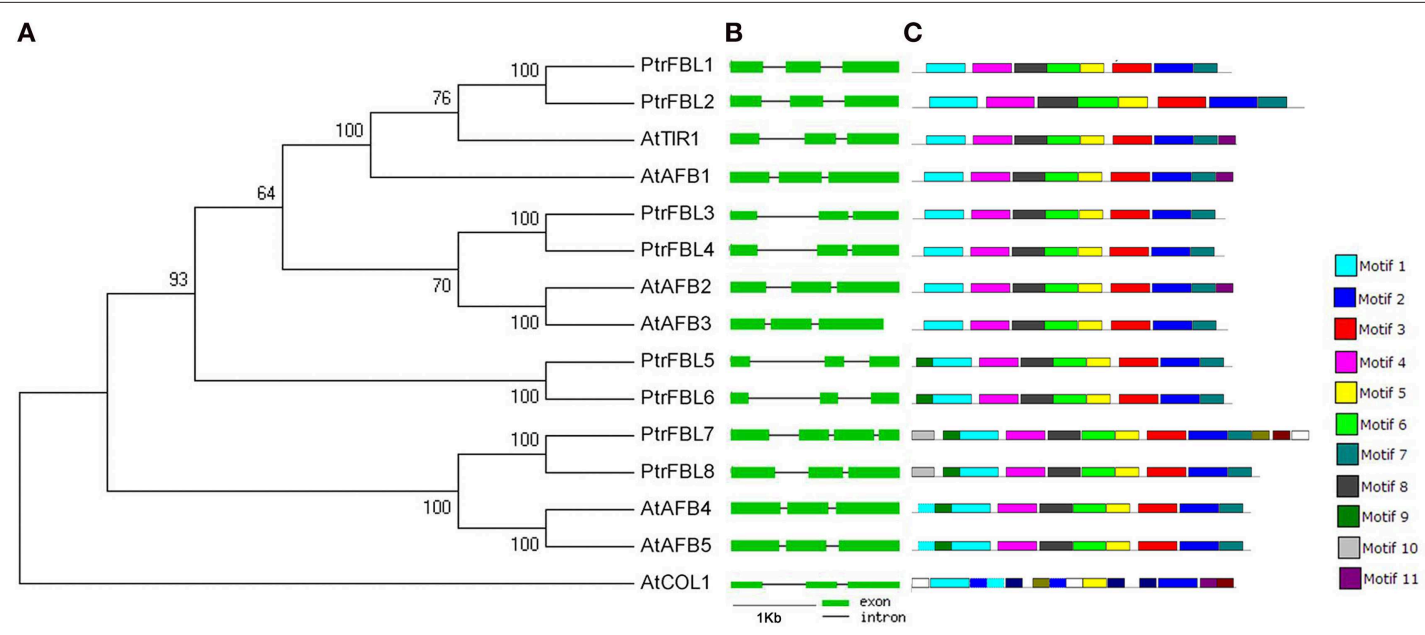

FIGURE 1 | Phylogenetic relationship, gene structures, and motif composition of TIR1 genes in A. thaliana (At), and $\boldsymbol{P}$. trichocarpa (Ptr). (A) A phylogenetic tree was constructed using MEGA 5 by the neighbor-joining (NJ) method with 1000 bootstrap replicates. The jasmonic acid receptor CONSTANS-LIKE 1 (AtCOL1) in Arabidopsis was used as an outgroup. (B) Exon/intron positions in the TIR1 receptors. Green boxes represent exons and black lines represent introns. (C) Schematic representation of conserved motifs (obtained using MEME) in TIR1 proteins. Different motifs are indicated by colored boxes. 
of 150-250 bp were designed using Primer 3 software (http:// frodo.wi.mit.edu/primer3/input.htm) (Table S1). The amplified fragments were confirmed using agarose gel electrophoresis. Real-time qRT-PCR was performed using the SYBR Premix Ex Taq II Kit (TaKaRa Dalian, Dalian, China) on a Roche LightCycle 480 Real-Time PCR System (Roche Applied Science, Germany) according to the manufacturer's instructions. Reactions were prepared in a total volume of $20 \mu \mathrm{l}$ containing $10 \mu \mathrm{l}$ of $2 \times$ SYBR Premix, $2 \mu \mathrm{l}$ of cDNA templates as prepared above and $1 \mu \mathrm{l}$ of each specific primer to a final concentration of $200 \mathrm{nM}$. The reactions were performed using the following conditions: initial denaturation step of $95^{\circ} \mathrm{C}$ for $30 \mathrm{~s}$, followed by 40 cycles of a two-step thermal profile of denaturation at $95^{\circ} \mathrm{C}$ for $10 \mathrm{~s}$ and annealing/extension at $60^{\circ} \mathrm{C}$ for $34 \mathrm{~s}$. To verify the specificity of each primer pair, a melting curve analysis was performed by increasing the temperature at a speed of $0.06^{\circ} \mathrm{C} / \mathrm{s}$ (five acquisitions per $1^{\circ} \mathrm{C}$ ) from 60 to $95^{\circ} \mathrm{C}$ at the end of each run. The threshold cycle values were the means of eight values from two biological repeats for each treatment with four technical replicates. The $U B Q$ gene was used as an internal control.

\section{NAA and Stress Treatment}

For NAA and polyethylene glycol (PEG) 6000 treatment, 4week-old $84 \mathrm{~K}$ seedlings were soaked in liquid Murashige and Skoog medium ( $\mathrm{pH}$ 5.8) under a $16 \mathrm{~h}$ light $/ 8 \mathrm{~h}$ dark regime at room temperature (mock pretreatment) for $12 \mathrm{~h}$, and then supplemented with $100 \mu$ M NAA (Sigma-Aldrich, St. Louis, MO, USA) for $10 \mathrm{~min}, 20 \mathrm{~min}, 30 \mathrm{~min}, 60 \mathrm{~min}$, and $360 \mathrm{~min}$, and with PEG6000 (5\%) for $0.5 \mathrm{~h}, 3 \mathrm{~h}, 6 \mathrm{~h}, 12 \mathrm{~h}$, and $24 \mathrm{~h}$. The leaves and stems were collected separately from treated seedlings at each time point and used for qRT-PCR analyses. The experiment was performed three times with four individual seedlings in each. The growth of 4-month-old over-expressing PtrFBL1 transgenic plants were measured on three representative lines (PtrFBL1-6, PtrFBL1-13, and PtrFBL1-15) with 12 cloned plants for each. The drought treatment was performed on the above transgenic plants and non-transgenic controls by stopping watering them in greenhouse for 6 days and re-watering, the plants were checked and photographed after 15 days. For measuring relative water content (RWC) of these poplars (PtrFBL1-6, PtrFBL1-13, and $84 \mathrm{~K}$ control) under the drought treatment, 3-month-old nonand transgenic plants were deprived of watering for 0,4 , and 6 days, and then the sixth leaf was detached from plants and weighed immediately to record their fresh weight (FW). The leaves were dipped in distiled water for $12 \mathrm{~h}$, then blotted briefly to remove the excess water and weighed to record the turgid weight (TW). The leaves were subjected to oven at $70{ }^{\circ} \mathrm{C}$ for $24 \mathrm{~h}$ to determine the dry weight (DW). The RWC was calculated using the equation: $\mathrm{RWC}=(\mathrm{FW}-\mathrm{DW}) \times 100 /(\mathrm{TW}-\mathrm{DW})$ as described previously (Negi et al., 2015). The experiments were performed with three biological replicates per treatment and the data from transgenic lines and non-transgenic controls were subjected to analysis of the variance (F-test, One-Way ANOVA) and the mean comparison among treatments was based on the least significant difference (LSD) test at the 5 and $1 \%$ level of significance using the package SPSS 17.0 (SPSS Inc, Chicago IL, USA).

\section{Results and Discussion}

\section{Characteristics of the Tir1 Gene Family in Arabidopsis and Populus}

We have identified eight TIR1 homologous genes (PtrFBLs) in $P$. trichocarpa (Table S2). All of the information on these eight PtrFBL genes, including gene names, locus IDs, genomic position, molecular weight (Mol. Wt), lengths and numbers of introns, are listed in Table S2. The ORF length of PtrFBL genes varied from 1716 bp (PtrFBL3) to 2172 bp (PtrFBL7), encoding polypeptides of 571-635 amino acids (aa), with a predicted molecular weight of $64.08-70.99 \mathrm{kDa}$ and a theoretical isoelectric point that ranged from 5.47 (PtrFBL8) to 7.35 (PtrFBL1) (Table S2).

To investigate the evolutionary relationship among TIR1/AFB receptors from Arabidopsis and $P$. trichocarpa, a phylogenetic tree was constructed, and the TIR1 proteins were classified into four clades that include the pairs of PtrFBL1/PtrFBL2 (AtTIR1/AtAFB1), PtrFBL3/PtrFBL4 (AtAFB2/AtAFB3), PtrFBL7/PtrFBL8 (AtAFB4/AtAFB5), and PtrFBL5/PtrFBL6 (Figure 1A), suggesting that these pairs are paralogs. Each of the Populus paralogous pairs shared $78-90 \%$ in sequence identity, but only had $40-78 \%$ identity to Arabidopsis TIR1/AFBs. Surprisingly, PtrFBL5 and PtrFBL6 had no homologous counterparts in Arabidopsis. We decided it may be more accurate to ascertain the evolutionary relationships using conserved domain sequences (Marchler-Bauer et al., 2009). Therefore, we constructed the phylogenetic tree with TIR1/AFBs motif sequences from Arabidopsis and Populus using the maximum likelihood method with 1000 bootstrap replicates (Figure S1). The resulted phylogenetic tree was consistent with the one generated based on the full-length protein sequences.

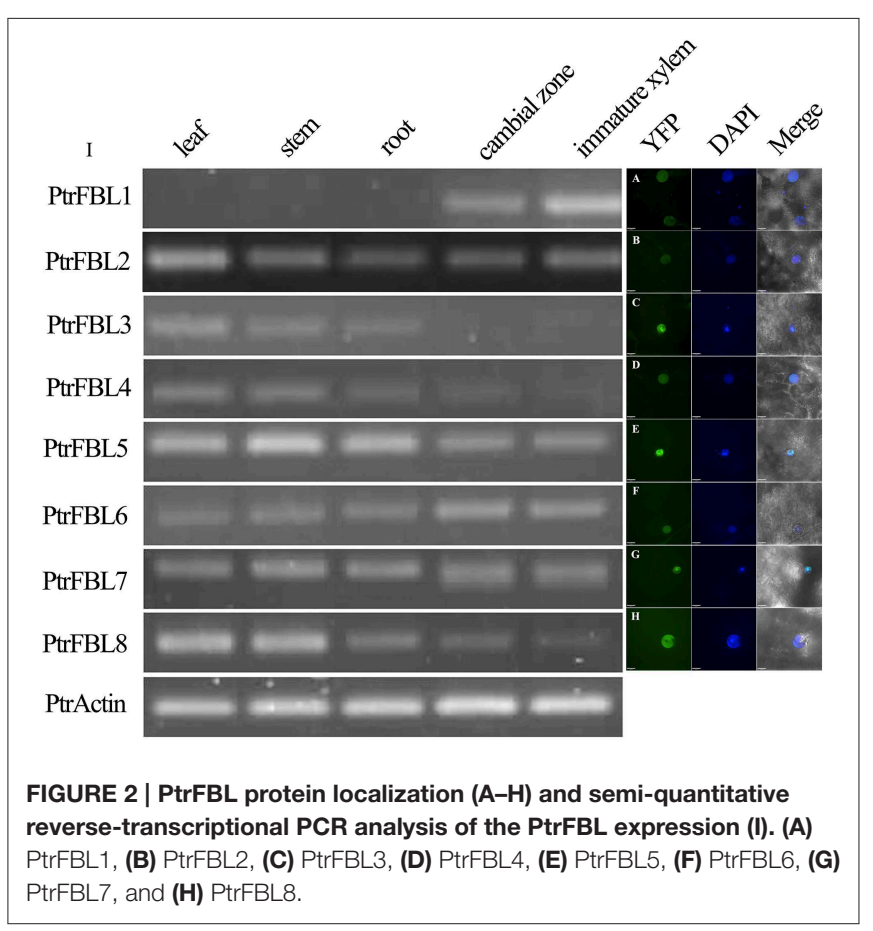


In many cases, the conservation of exon/intron organization or gene structure in paralogous genes was high and could be used to reveal their evolutionary relationship (Sánchez et al., 2003). We, therefore, analyzed the exon/intron organization in the coding sequence of each PtrFBL in Populus and TIR1/AFB in Arabidopsis, which showed that each paralogous pair shared similar gene structures in terms of intron number or exon length, with the exception of PtrFBL7 harboring one extra intron (Figure 1B, Table S2). In addition, MEME was employed to analyze motif distributions in 14 TIR1 proteins and 11 individual motifs found in Populus and Arabidopsis TIR1 receptors. Of these, motifs $3,4,6,7$, and 8 were found to be specific to the two species (Figure 1C, Table S3). Most of the closely related members have common motif compositions, suggesting possible functional similarities among these TIR1 proteins (Figure 1C).

Studies have found that gene duplication events are the basic contributors to evolutionary momentum (Bowers et al., 2003). The co-linear arrangements of the adjacent genes, as well as the four gene pairs (PtrFBL1/PtrFBL2, PtrFBL3/PtrFBL4, PtrFBL5/PtrFBL6, and PtrFBL7/PtrFBL8), further revealed that these paralogous pairs came from the genome duplication in Populus (Figure S2). The chromosomal mapping of the gene loci showed that all eight PtrFBL genes were distributed on 7 of 19 chromosomes, with two PtrFBL genes located on chromosome 2, and one each on chromosome 1, 4, 5, 11, 14, and 17 (Figure S3). PtrFBL1/PtrFBL2 and PtrFBL7/PtrFBL8 were distributed in the corresponding duplicated segments of the chromosomes (Tuskan et al., 2006), while PtrFBL3/PtrFBL4 and PtrFBL5/PtrFBL6 did not appear in the corresponding duplicated regions (Figure S3). However, only two such pairs (AtAFB2/AtAFB3 and AtAFB4/AtAFB5) were found in Arabidopsis (Figure S2). Genome duplication events were thought to occur frequently during organismal evolution (Mehan et al., 2004). It is suggested that the Populus genome has experienced at least two genomewide duplication events (eurosid and salicoid), followed by a series of chromosomal reorganizations involving reciprocal
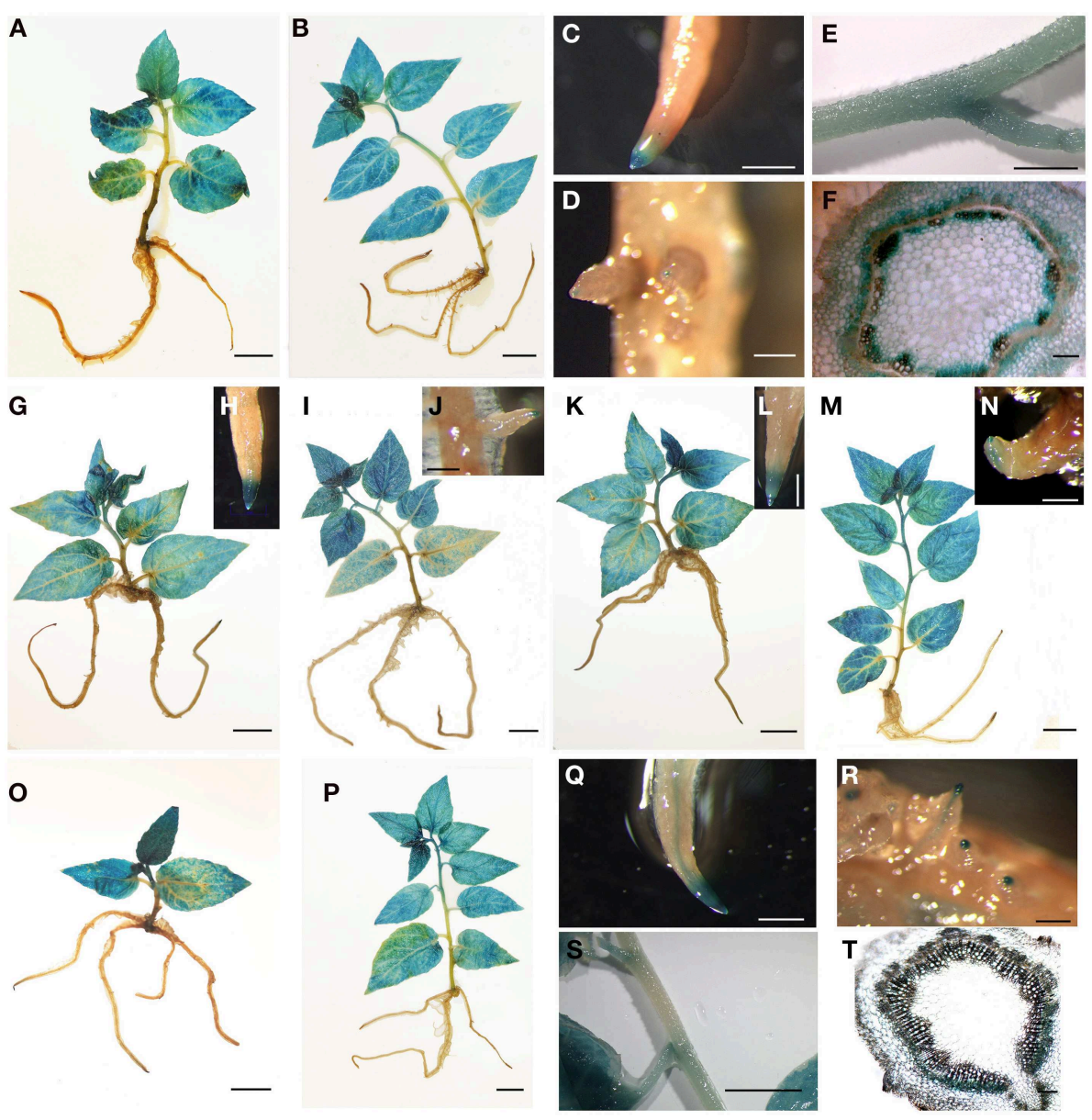

FIGURE 3 | GUS assay of transgenic poplars driven by PtrFBL promoters. (A-F) $P_{P t r F B L 1}:: G U S$ : (A) 2-week-old seedling, (B) 4-week-old seedling, (C) root tip, (D) lateral root tip, and (E,F) GUS expressed mainly in stem; (G-J) $P_{\text {PtrFBL4 }}:: G U S:$ (G) 2-week-old seedling, (I) 4-week-old seedling, (H) root tip and (J) lateral root

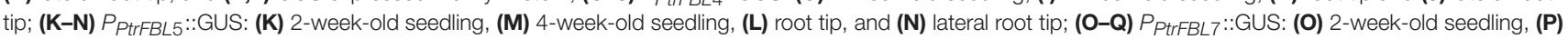
4-week-old seedling, (Q) root tip and (R) lateral root tip; (S,T) GUS is expressed mainly in stems near the petiole. Bars, $1 \mathrm{~cm}(\mathbf{A}-\mathbf{C}, \mathbf{E}, \mathbf{G}, \mathbf{I}, \mathbf{K}, \mathbf{M}, \mathbf{O}, \mathbf{P}, \mathbf{S}), 2 \mathrm{~mm}$ (D,H,J,L,N,Q,R), $0.1 \mathrm{~mm}(\mathbf{F}, \mathbf{T})$. 
tandem/terminal fusions and translocations (Tuskan et al., 2006). Therefore, recent duplication events in Populus could have led to the creation of the four PtrFBL pairs, and the latter could have undergone intensive genome rearrangements afterwards, during which the PtrFBL3/PtrFBL4 and PtrFBL5/PtrFBL6 segments were not maintained in their corresponding duplicated regions (Figures S2, S3).

All the PtrFBLs were predicted to be localized in the nucleus using WoLF PSORT (Table S2). To confirm this, these proteins, fused with YFP at the C-terminus, were transiently expressed in tobacco leaf epidermal cells, and the fusion proteins were observed in the nucleus as indicated by co-localization with DAPI signals (Figures 2A-H). This was consistent with the nuclear localizations of TIR1/AFB proteins in Arabidopsis (Dharmasiri et al., 2005a; Tan et al., 2007). The conserved organelle localization of PtrFBLs implied their conserved functions, such as the regulation of the cellular auxin response.

\section{Expression Patterns of PtrFBLs}

The expression patterns of genes can provide useful clues to the functions of their corresponding proteins. The expression of PtrFBLs in the leaves, stems, roots, cambial zones and immature xylem of Populus tomentosa Carr. was analyzed by semi-quantitative RT-PCR (Figure 2I). PtrFBL1 was mainly expressed in the cambial zone and immature xylem, while
PtrFBL3 and 4 were mainly expressed in the leaves, stems and roots. On the contrary, PtrFBL2, 3, and 8 were enriched in the leaves. The expression levels of PtrFBL1 and 6 in the cambial zone were higher than in the other tissues, while PtrFBL5 and 8 were found at higher levels in young stems but at lower levels in secondary vascular tissues. The PtrFBL3/PtrFBL4 and PtrFBL7/PtrFBL8 pairs, which had similar promoter sequences based on the phylogenetic analysis of $2.5 \mathrm{~kb}$ upstream sequences of the PtrFBL genes (Figure S4), exhibited similar expression patterns. The analysis of the RNA-seq data from different Populus vegetative tissues (unpublished data) further confirmed these PtrFBLs expression patterns (Figure S5).

To further confirm the expression patterns of the PtrFBL genes, four PtrFBLs with different expression patterns in different tissues were selected for analysis using a promoter::GUS assay. The transgenic poplars $(P$. alba $\times P$. glandulosa) with $P_{\text {PtrFBL1 }}:$ GUS, $P_{\text {PtrFBL } 4:: G U S,} P_{\text {PtrFBL5 }}:$ GUS and $P_{\text {PtrFBL7 }}::$ GUS were obtained, and GUS assays of whole plants were performed. Similar to the expression observed in Arabidopsis, expression levels of these PtrFBLs were high in the young tissues but low in the older tissues (Figure 3). However, the expression levels varied in different organs and tissues. Except in young leaves, primary stems, roots and lateral root tips, PtrFBL1 was found to mainly express in vascular tissues abaxial and adaxial to the cambium (Figures 3E,F), in accordance with semiquantitative PCR results (Figure 2I). PtrFBL7 was also observed

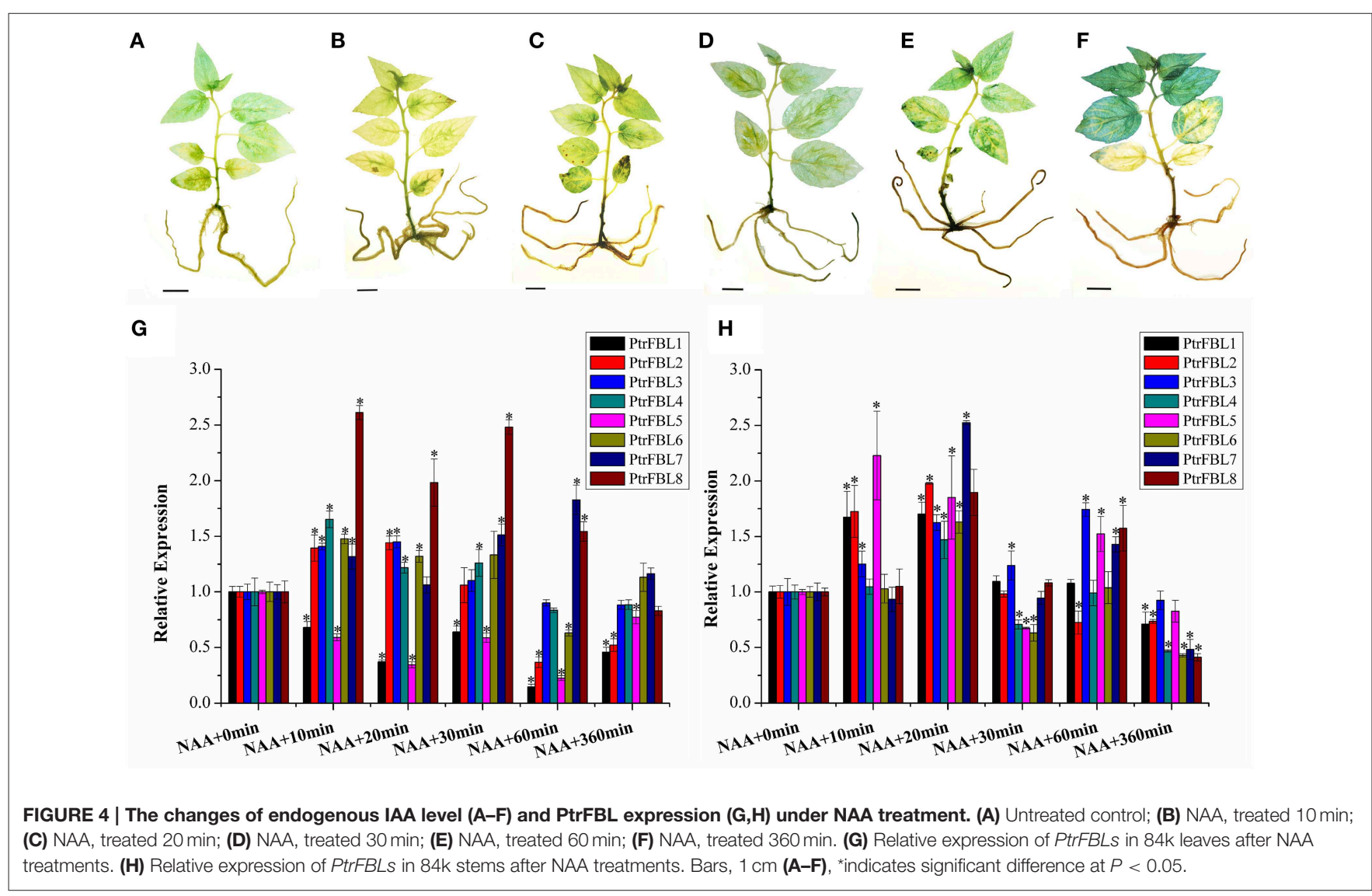


to be highly expressed in the cambial zone (Figure 2I), but closer observations revealed the expression was restricted to the cambium in the stem sections (Figures 3S,T), in compensation to the area of vascular tissues uncovered by the PtrFBL1 expression. However, although GUS staining of the whole

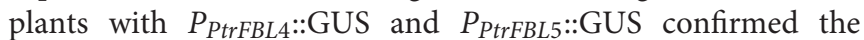
general expression patterns of these genes (Figures 3G-N), the expression was present in root tips (Figures 3H,J,L,N) and absent in root vascular tissues. In Arabidopsis, the AtAFB1::GUS protein was abundant throughout the Arabidopsis seedling, while the accumulations of AtTIR1, AtAFB2, and AtAFB3 proteins were highly restricted to growing tissues, including root tips, leaf primordia, and shoot meristems (Dharmasiri et al., 2005b; Parry et al., 2009). Therefore, poplar PtrFBLs exhibited more diversified expression patterns in different tissues. For instance, the differential expression of PtrFBL1 and PtrFBL7 was observed in secondary vascular tissues (Figure 3). In addition, these two genes may play differential roles in the maintenance of cambial activity and its differentiation into vascular tissues by mediating different auxin signaling. This phenomenon could not be observed in Arabidopsis because it lacks secondary vascular tissues.

\section{Low NAA Responses of PtrFBLs}

To reveal whether PtrFBL genes respond to auxin, we analyzed the expression profiles of PtrFBLs in poplar treated with NAA. We used poplar lines with DR5::GUS as an auxin marker (Liu et al., 2014a) to monitor the response of auxin in whole seedlings under NAA treatment (Figures $\mathbf{4 A - F}$ ), which showed that the endogenous auxin response decreased at an early stage then increased gradually. The PtrFBL transcripts in general were not significantly affected by NAA treatment as quantified by qRTPCR (Figures 4G,H). The limited responses of PtrFBLs to NAA treatment in Populus in this study and in Arabidopsis (Parry et al., 2009) indicated that FBLs were not very sensitive to auxin. However, a considerably lower expression levels of PtrFBL1 and 7 , which showed unique expression patterns in the cambial zone (Figure 3), were observed in stems after NAA treatment

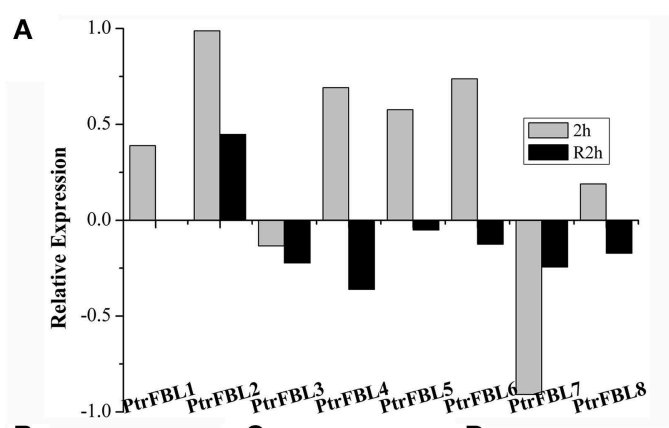

B

C

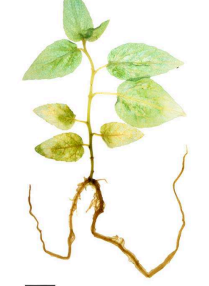

H

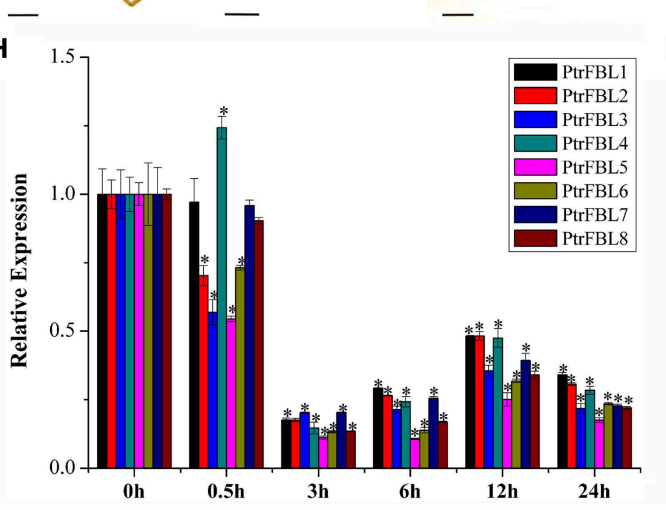

E

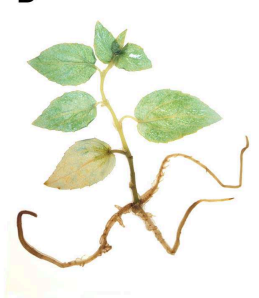

I

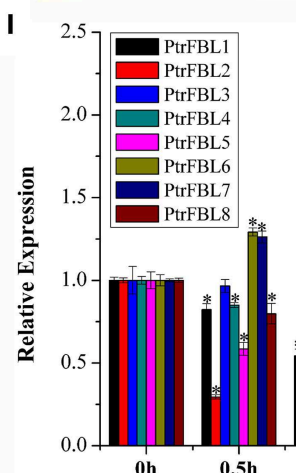

$\mathbf{F}$
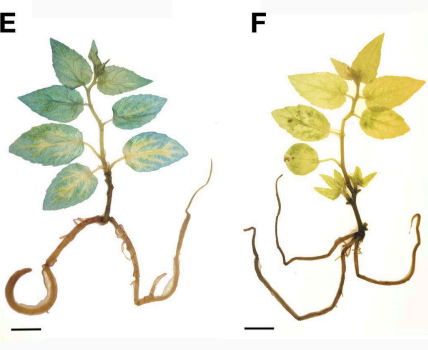

G

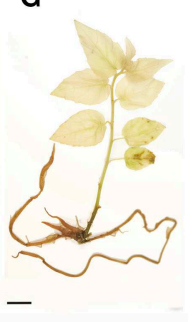

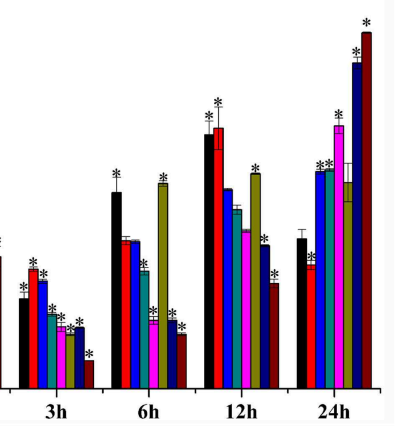

FIGURE 5 | Expression profiles of PtrFBL genes under heat and drought treatments. (A) Heat map showing eight PtrFBL genes under heat stress. 2 h: $2 \mathrm{~h}$ of $37^{\circ} \mathrm{C}$ heat treatment; R2h: $2 \mathrm{~h}$ recovery after $37^{\circ} \mathrm{C}$ heat treatment. Endogenous IAA levels expressed in DR5-GUS plants under PEG6000 stress (B-G); (B) $0 \mathrm{~h}$, (C) 0.5 h, (D) $3 \mathrm{~h}, \mathbf{( E )} 6 \mathrm{~h}, \mathbf{( F )} 12 \mathrm{~h}$, and (G) $24 \mathrm{~h}$ treatments; (H,I) Expression profiles of eight PtrFBL genes under PEG6000 stress; (H) in $84 \mathrm{k}$ leaf; (I) in $84 \mathrm{k}$ stems. Bars, $1 \mathrm{~cm}$ (B-G), *indicates significant difference at $P<0.05$. 
(Figures 4G,H). This may suggest their specific functions in vascular tissue development.

\section{Differential Stress Responses of PtrFBLs}

Temperature was an important factor that limits the growth, development, and geographical distribution of plants (Nguyen et al., 2009), and high temperatures altered auxin responses by changing auxin synthesis (Franklin et al., 2011). We investigated the expression of the PtrFBL genes based on our RNA-seq data (unpublished) from heat-treated seedlings by transferring them from 25 to $37^{\circ} \mathrm{C}$ and incubating for $2 \mathrm{~h}$. The results showed the different responses of PtrFBL members under heat stress (Figure 5A). Previous studies in Arabidopsis found that the quadruple mutant tir1/afb1/afb2/afb3 exhibited a delayed response but also a strongly reduced hyponastic growth response amplitude after the initiation of the heat treatment (van Zanten et al., 2009). Therefore, FBLs are among the primary factors involved in heat stress response.

Drought treatment with PEG6000 had significant effects on the response of auxin as indicated by the DR5::GUS line (Figures 5B-G). Endogenous auxin increased in the early stage, but decreased in the later stage of the treatment. Relatively low expression levels of all the PtrFBL genes in leaves and stems were observed in the first $3 \mathrm{~h}$, but they recovered almost to the same, or higher, levels in the stems. However, they remained low in the leaves $6 \mathrm{~h}$ after treatment compared with the untreated plants (Figures $\mathbf{5 H}, \mathbf{I}$ ). The differences in the expression levels of PtrFBLs in leaves and stems suggest their regulatory roles in reprogramming plant development to cope with drought stress by lowering the auxin signaling in leaves while strengthening it in stems.

\section{Over-expression of PtrFBL1 Affected the Growth and Drought Tolerance of Transgenic Plants}

To investigate the role of PtrFBL1 in stem development, we successfully generated $84 \mathrm{~K}$ transgenic lines constitutively overexpressing PtrFBL1 under the control of the $35 \mathrm{~S}$ promoter. Three representative transgenic lines with relative expression over 14-fold were used for further analysis (Figures 6A,B). We observed apparent phenotypic changes in height and diameter of the transgenic plants (Figures 6A,C), which were significantly higher than that of the non-transgenic controls $(P=0.015$ and $P=0.006$, respectively). However, we found that the transgenic plants exhibited decreased tolerance under drought treatment (Figures 6D,E). RWC was a measure of plant water status and used as a meaningful index of water stress tolerance (Negi et al., 2015). In our study, the RWC values obtained from plants under drought stress showed that the transgenic lines retained less water than the non-transgenic plants (Table 1). The difference was significant for transgenic line PtrFBL1-13 at 4 days $(P=0.011)$ and for both lines at 6 days $(P=0.001)$ after the deprival of watering. This indicated that PtrFBL1 played a key role in the balance of plant growth and tolerance. Although the roles of TIR1 in many developmental processes in Arabidopsis have been well documented (Parry et al., 2009), the effects of its homolog PtrFBL1 in poplar on the stem growth and tolerance were first found in this study.
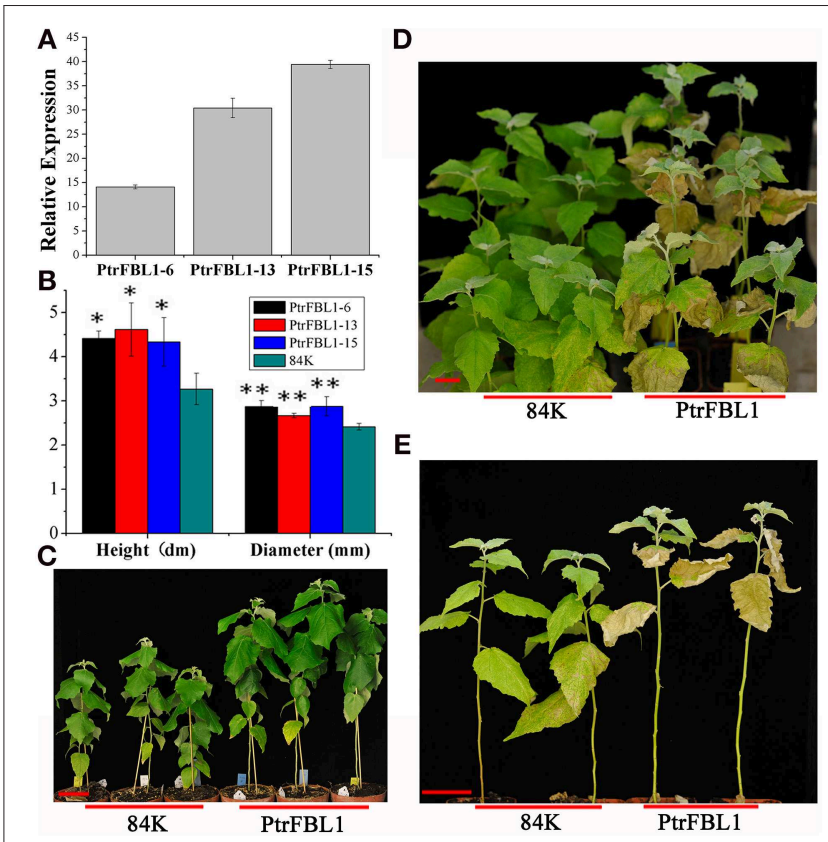

FIGURE 6 | The growth and drought tolerance of PtrFBL1

over-expressed poplars. (A) Phenotypic changes in stem growth of over-expressing PtrFBL1; (B) Relative expression for over-expressing PtrFBL1; (C) The height and diameters of non- and transgenic lines; (D,E) Phenotypic changes of the PtrFBL1 over-expressed plants after drought treatment for 6 days and the photos were taken 15 days after rewatering. Bars, $5 \mathrm{~cm}$ (A,D,E), *indicates significant difference at $P<0.05$, and ${ }^{* *}$ indicates extremely significant difference at $P<0.01$.

TABLE 1 | The RWC values of the leaves at 0,4 , and 6 days after the deprival of watering.

\begin{tabular}{lccc}
\hline Line & 0 day & 4 days & 6 days \\
\hline PtrFBL1-6 & $90.113 \pm 0.0827$ & $91.237 \pm 2.138$ & $89.634 \pm 0.213^{\star *}$ \\
PtrFBL1-13 & $90.927 \pm 1.173$ & $88.906 \pm 1.560^{\star}$ & $91.220 \pm 0.949^{\star *}$ \\
Non-transgenic 84k & $91.292 \pm 1.107$ & $93.680 \pm 0.630$ & $93.787 \pm 0.639$
\end{tabular}

Value $=$ mean $\pm S D$; ${ }^{*}$ significance at $P \leq 0.05$; ${ }^{* *}$ significance at $P \leq 0.01$.

The evolutionary features of TIR1/AFB proteins across species revealed considerable conservation of sequence and structure, as well as evolutionary diversity following the genome duplication events and intensive segmental recombination. Expression pattern analysis, however, showed that different members of PtrFBLs displayed distinctive expression patterns in different Populus organs and tissues. Interestingly, PtrFBL1 was found to express in vascular tissues abaxial and adaxial to the cambium, while PtrFBL7 was only observed to be highly expressed in the cambial zone, suggesting their different roles in the development of secondary vascular systems in Populus. In addition, most tested genes were changed by exogenous treatments with auxin, heat and drought, and exhibited varying dynamic expression patterns. The further functional analysis of PtrFBL1 has confirmed its role in the stem growth as well as 
in balancing the growth and tolerance under stress conditions. Our data imply that different PtrFBL genes may be involved in particular developmental processes, which deserves further characterization of their roles in the development of woody plants.

\section{Author Contributions}

WS carried out all the analyses and drafted the manuscript. YL helped in collecting Populus materials. YG performed the NAA experiments, and contributed to the results and discussion sections of the manuscript. HZ helped with transgenic seedling identification, total RNA extraction and RT-PCR analyses. JZ helped with data analyses of bioinformatics, as well as tissue and heat stress experiments. SZ and ML contributed intellectually to all aspects of this research and helped in finalizing the manuscript. All authors read and approved the final manuscript.

\section{Acknowledgments}

This work was supported by the National Key Basic Research Program of China (2012CB114500) and the National High Technology Research and Development Program of China (2013AA102702) to ML.

\section{References}

Bailey, T. L., Boden, M., Buske, F. A., Frith, M., Grant, C. E., Clementi, L., et al. (2009). MEME SUITE: tools for motif discovery and searching. Nucleic Acids Res. 37 (Suppl. 2), W202-W208. doi: 10.1093/nar/gkp335

Bowers, J. E., Chapman, B. A., Rong, J., and Paterson, A. H. (2003). Unravelling angiosperm genome evolution by phylogenetic analysis of ch romosomal duplication e vents. Nature 422, 433-438. doi: 10.1038/nature01521

Calderón Villalobos, L. I., Lee, S., De Oliveira, C., Ivetac, A., Brandt, W., Armitage, L., et al. (2012). A combinatorial TIR1/AFB-Aux/IAA co-receptor system for differential sensing of auxin. Nat. Chem. Biol. 8, 477-485. doi: 10.1038/nchembio. 926

Chapman, E. J., and Estelle, M. (2009). Cytokinin and auxin intersection in root meristems. Genome Biol. 10:210. doi: 10.1186/gb-2009-10-2-210

Chen, Z., Hu, L., Han, N., Hu, J., Yang, Y., Xiang, T., et al. (2015). Overexpression of a miR393-resistant form of transport inhibitor response Protein 1 (mTIR1) enhances salt tolerance by increased osmoregulation and $\mathrm{Na}^{+}$exclusion in Arabidopsis thaliana. Plant Cell Physiol. 56, 73-83. doi: 10.1093/pcp/pcu149

Dharmasiri, N., Dharmasiri, S., and Estelle, M. (2005a). The F-box protein TIR1 is an auxin receptor. Nature 435, 441-445. doi: 10.1038/nature03543

Dharmasiri, N., Dharmasiri, S., Weijers, D., Lechner, E., Yamada, M., Hobbie, L., et al. (2005b). Plant development is regulated by a family of auxin receptor $\mathrm{F}$ box proteins. Dev. Cell 9, 109-119. doi: 10.1016/j.devcel.2005.05.014

Dreher, K. A., Brown, J., Saw, R. E., and Callis, J. (2006). The Arabidopsis Aux/IAA protein family has diversified in degradation and auxin responsiveness. Plant Cell 18, 699-714. doi: 10.1105/tpc.105.039172

$\mathrm{Du}$, J., and Groover, A. (2010). Transcriptional regulation of secondary growth and wood formation. J. Integr. Plant Biol. 52, 17-27. doi: 10.1111/j.17447909.2010.00901.x

Franklin, K. A., Lee, S. H., Patel, D., Kumar, S. V., Spartz, A. K., Gu, C., et al. (2011). Phytochrome-interacting factor 4 (PIF4) regulates auxin biosynthesis at high temperature. Proc. Natl. Acad. Sci. U.S.A. 108, 20231-20235. doi: 10.1073/pnas.1110682108

\section{Supplementary Material}

The Supplementary Material for this article can be found online at: http://journal.frontiersin.org/article/10.3389/fpls.2015. 00719

Table S1 | Primer sequences for PtrFBLs Promoter (P), Subcellular (S), semi-quantitative PCR (RT), and quantitative PCR (qRT).

Table S2 | TIR1 genes families in Arabidopsis and Populus.

Table S3 | The conserved motifs of TIR1 proteins in Arabidopsis and Populus.

Figure S1 | Phylogenetic analysis of the TIR1 family of genes from Arabidopsis and $P$. trichocarpa using full-length nucleotide sequences.

Figure S2 | Gene duplication relationships in the TIR1 gene family of Arabidopsis and Populus. Blue lines represent the other anchor gene pairs in the region, and the red line represents the query locus.

Figure S3 | Genomic localizations of the full-length PtrFBL genes. Eight PtrFBL genes were mapped to seven linkage groups (LG). Segmentally duplicated homologous blocks are indicated using the same color. The black lines represents a $10-\mathrm{Mb}$ chromosomal distance.

Figure S4 | Phylogenetic relationships of FBL gene promoters in P. trichocarpa. A multiple alignment of the sequences $2.5 \mathrm{~kb}$ upstream of the PtrFBL genes was executed using ClustalX 2.1 and a phylogenetic tree was constructed using MEGA 5 by the neighbor-joining (NJ) method with 1000 bootstrap replicates.

Figure S5 | Heat map showing the expression of eight PtrFBL genes in vegetative tissues (YL, young leaves; ML, mature leaves; PS, primary stem; SS, secondary stem; and $\mathrm{R}$, roots).

Fuchs, M., van Bel, A. J., and Ehlers, K. (2010). Season-associated modifications in symplasmic organization of the cambium in Populus nigra. Ann. Bot. 105, 375-387. doi: 10.1093/aob/mcp300

Gagne, J. M., Downes, B. P., Shiu, S.-H., Durski, A. M., and Vierstra, R. D. (2002). The F-box subunit of the SCF E3 complex is encoded by a diverse superfamily of genes in Arabidopsis. Proc. Natl. Acad. Sci. U.S.A. 99, 11519-11524. doi: 10.1073/pnas.162339999

Greenham, K., Santner, A., Castillejo, C., Mooney, S., Sairanen, I., Ljung, K., et al. (2011). The AFB4 auxin receptor is a negative regulator of auxin signaling in seedlings. Curr. Biol. 21, 520-525. doi: 10.1016/j.cub.2011. 02.029

Guerra, D., Crosatti, C., Khoshro, H. H., Mastrangelo, A. M., Mica, E., Mazzucotelli, E., et al. (2015). Post-transcriptional and post-translational regulations of drought and heat response in plants: a spider's web of mechanisms. Front. Plant Sci. 6:57. doi: 10.3389/fpls.2015.00057

Havens, K. A., Guseman, J. M., Jang, S. S., Pierre-Jerome, E., Bolten, N., Klavins, E., et al. (2012). A synthetic approach reveals extensive tunability of auxin signaling. Plant Physiol. 160, 135-142. doi: 10.1104/pp.112. 202184

Hu, B., Jin, J., Guo, A.-Y., Zhang, H., Luo, J., and Gao, G. (2015). GSDS 2.0: an upgraded gene feature visualization server. Bioinformatics 31, 1296-1297. doi: 10.1093/bioinformatics/btu817

Hu, Z., Keçeli, M. A., Piisilä, M., Li, J., Survila, M., Heino, P., et al. (2012). F-box protein AFB4 plays a crucial role in plant growth, development and innate immunity. Cell Res. 22, 777-781. doi: 10.1038/cr. 2012.12

Jurado, S., Díaz-Triviño, S., Abraham, Z., Manzano, C., Gutierrez, C., and del Pozo, C. (2008). SKP2A, an F-box protein that regulates cell division, is degraded via the ubiquitin pathway. Plant J. 53, 828-841. doi: 10.1111/j.1365313X.2007.03378.x

Kalluri, U. C., DiFazio, S. P., Brunner, A. M., and Tuskan, G. A. (2007). Genomewide analysis of Aux/IAA and ARF gene families in Populus trichocarpa. BMC Plant Biol. 7:59. doi: 10.1186/1471-2229-7-59 
Kramer, E. M., and Bennett, M. J. (2006). Auxin transport: a field in flux. Trends Plant Sci. 11, 382-386. doi: 10.1016/j.tplants.2006.06.002

Larkin, M. A., Blackshields, G., Brown, N. P., Chenna, R., McGettigan, P. A., McWilliam, H., et al. (2007). Clustal W and Clustal X version 2.0. Bioinformatics 23, 2947-2948. doi: 10.1093/bioinformatics/btm404

Lau, S., Shao, N., Bock, R., Jürgens, G., and De Smet, I. (2009). Auxin signaling in algal lineages: fact or myth? Trends Plant Sci. 14, 182-188. doi: $10.1016 /$ j.tplants.2009.01.004

Liu, B., Wang, L., Zhang, J., Li, J., Zheng, H., Chen, J., et al. (2014a). WUSCHELrelated Homeobox genes in Populus tomentosa: diversified expression patterns and a functional similarity in adventitious root formation. BMC Genomics 15:296. doi: 10.1186/1471-2164-15-296

Liu, B., Zhang, J., Wang, L., Li, J., Zheng, H., Chen, J., et al. (2014b). A survey of Populus PIN-FORMED family genes reveals their diversified expression patterns. J. Exp. Bot. 65, 2437-2448. doi: 10.1093/jxb/eru129

Locascio, A., Roig-Villanova, I., Bernardi, J., and Varotto, S. (2014). Current perspectives on the hormonal control of seed development in Arabidopsis and maize: a focus on auxin. Front. Plant Sci. 5:412. doi: 10.3389/fpls.2014.00412

Ludwig-Müller, J. (2011). Auxin conjugates: their role for plant development and in the evolution of land plants. J. Exp. Bot. 62, 1757-1773. doi: $10.1093 /$ jxb/erq412

Marchler-Bauer, A., Anderson, J. B., Chitsaz, F., Derbyshire, M. K., DeWeeseScott, C., Fong, J. H., et al. (2009). CDD: specific functional annotation with the Conserved Domain Database. Nucleic Acids Res. 37, D205-D210. doi: 10.1093/nar/gkn845

Mehan, M. R., Freimer, N. B., and Ophoff, R. A. (2004). A genome-wide survey of segmental duplications that mediate common human genetic variation of chromosomal architecture. Hum. Genomics 1:335. doi: 10.1186/1479-7364-15-335

Negi, N. P., Shrivastava, D. C., Sharma, V., and Sarin, N. B. (2015). Overexpression of CuZnSOD from Arachis hypogaea alleviates salinity and drought stress in tobacco. Plant Cell Rep. 34, 1109-1126. doi: 10.1007/s00299-015-1770-4

Nguyen, H. T., Leipner, J., Stamp, P., and Guerra-Peraza, O. (2009). Low temperature stress in maize (Zea mays L.) induces genes involved in photosynthesis and signal transduction as studied by suppression subtractive hybridization. Plant Physiol. Biochem. 47, 116-122. doi: 10.1016/j.plaphy.2008.10.010

Parry, G., Calderon-Villalobos, L. I., Prigge, M., Peret, B., Dharmasiri, S., Itoh, H., et al. (2009). Complex regulation of the TIR1/AFB family of auxin receptors. Proc. Natl. Acad. Sci. U.S.A. 106, 22540-22545. doi: 10.1073/pnas.0911967106

Peer, W. A. (2013). From perception to attenuation: auxin signalling and responses. Curr. Opin. Plant Biol. 16, 561-568. doi: 10.1016/j.pbi.2013.08.003

Remington, D. L., Vision, T. J., Guilfoyle, T. J., and Reed, J. W. (2004). Contrasting modes of diversification in the Aux/IAA and ARF gene families. Plant Physiol. 135, 1738-1752. doi: 10.1104/pp.104.039669

Robert, S., Kleine-Vehn, J., Barbez, E., Sauer, M., Paciorek, T., Baster, P., et al. (2010). ABP1 mediates auxin inhibition of clathrin-dependent endocytosis in Arabidopsis. Cell 143, 111-121. doi: 10.1016/j.cell.2010.09.027
Salehin, M., Bagchi, R., and Estelle, M. (2015). SCFTIR1/AFB-based auxin perception: mechanism and role in plant growth and development. Plant Cell 114:133744. doi: 10.1105/tpc.114.133744

Sánchez, D., Ganfornina, M. D., Gutiérrez, G., and Marín, A. (2003). Exonintron structure and evolution of the Lipocalin gene family. Mol. Biol. Evol. 20, 775-783. doi: 10.1093/molbev/msg079

Tamura, K., Peterson, D., Peterson, N., Stecher, G., Nei, M., and Kumar, S. (2011). MEGA5: molecular evolutionary genetics analysis using maximum likelihood, evolutionary distance, and maximum parsimony methods. Mol. Biol. Evol. 28, 2731-2739. doi: $10.1093 / \mathrm{molbev} / \mathrm{msr} 121$

Tan, X., Calderon-Villalobos, L. I. A., Sharon, M., Zheng, C., Robinson, C. V., Estelle, M., et al. (2007). Mechanism of auxin perception by the TIR1 ubiquitin ligase. Nature 446, 640-645. doi: 10.1038/nature 05731

Tuskan, G. A., Difazio, S., Jansson, S., Bohlmann, J., Grigoriev, I., Hellsten, U., et al. (2006). The genome of black cottonwood, Populus trichocarpa (Torr. \& Gray). Science 313, 1596-1604. doi: 10.1126/science. 1128691

van Zanten, M., Voesenek, L. A., Peeters, A. J., and Millenaar, F. F. (2009). Hormone-and light-mediated regulation of heat-induced differential petiole growth in Arabidopsis. Plant Physiol. 151, 1446-1458. doi: 10.1104/pp.109.144386

Vidal, E. A., Araus, V., Lu, C., Parry, G., Green, P. J., Coruzzi, G. M., et al. (2010). Nitrate-responsive miR393/AFB3 regulatory module controls root system architecture in Arabidopsis thaliana. Proc. Natl. Acad. Sci. U.S.A. 107, 4477-4482. doi: 10.1073/pnas.0909571107

Walsh, T. A., Neal, R., Merlo, A. O., Honma, M., Hicks, G. R., Wolff, K., et al. (2006). Mutations in an auxin receptor homolog AFB5 and in SGT1b confer resistance to synthetic picolinate auxins and not to 2, 4-dichlorophenoxyacetic acid or indole-3-acetic acid in Arabidopsis. Plant Physiol. 142, 542-552. doi: 10.1104/pp.106.085969

Woodward, A. W., and Bartel, B. (2005). Auxin: regulation, action, and interaction. Ann. Bot. 95, 707-735. doi: 10.1093/aob/mci083

Yu, H., Moss, B. L., Jang, S. S., Prigge, M., Klavins, E., Nemhauser, J. L., et al. (2013). Mutations in the TIR1 auxin receptor that increase affinity for auxin/indole-3acetic acid proteins result in auxin hypersensitivity. Plant Physiol. 162, 295-303. doi: $10.1104 /$ pp.113.215582

Conflict of Interest Statement: The authors declare that the research was conducted in the absence of any commercial or financial relationships that could be construed as a potential conflict of interest.

Copyright (c) $2015 \mathrm{Shu}, \mathrm{Liu}, \mathrm{Guo}, \mathrm{Zhou}$, Zhang, Zhao and Lu. This is an open-access article distributed under the terms of the Creative Commons Attribution License (CC $B Y)$. The use, distribution or reproduction in other forums is permitted, provided the original author(s) or licensor are credited and that the original publication in this journal is cited, in accordance with accepted academic practice. No use, distribution or reproduction is permitted which does not comply with these terms. 